\title{
Model Order Reduction of Dynamical Systems: An Approach to Investigate Real and Complex Poles
}

\author{
Moomal Bukhari ${ }^{1}$, Mukhtar Ullah ${ }^{1,2}$, Mukhtar Ali $^{1}$, Jamshed Iqbal ${ }^{1,3}$ \\ ${ }^{1}$ Department of Electrical Engineering, \\ FAST National University of Computer and Emerging Sciences, Islamabad, Pakistan \\ ${ }^{2}$ Department of Electrical Engineering, MY University, Islamabad, Pakistan \\ ${ }^{3}$ Electrical and Computer Engineering Department, University of Jeddah, Saudi Arabia \\ moomal.bukhari@nu.edu.pk,mukhtar.ullah@nu.edu.pk,mukhtar_ali24@yahoo.com,jmiqbal@uj.edu.sa
}

\begin{abstract}
The process of model order reduction facilitates analysis and realization of complex systems while marinating the essential properties of the system and input/output behavior. This research proposes a novel method for model order reduction. The novelty of the proposed method lies in addressing the limitations of dimensionless representation and pole-zero dominance. Both real and complex poles are treated for order reduction. The reduction of dimensionless systems, which considers real poles only, in the field of controls has not been considerably investigated. Even MATLAB has not completely exploited it. This work also investigates the augmentation of dimensionless systems and singular values based order reduction technique. For systems with complex poles, pole-to-pole and poleto-zero ratios are considered for the reduction. The performance of the proposed method is demonstrated with simulated examples of multiple systems. The results indicate that the error between the unit step responses of the reduced and the high order model is less than $5 \%$. This research may find potential in various applications involving model order reduction including but not limited to; circuit simulation, micro-electromechanical systems, avionics and structural dynamics.
\end{abstract}

Index Terms - Model order reduction, Hankel singular values, balanced realization, controllability, observability.

\section{INTRODUCTION}

Building and analyzing quantitative models of physical systems are pivotal in control engineering. Analysis of a high order system is a challenging task [1]. Model order reduction tools play an important role in both research and industrial applications to simplify the analysis process, the realization process, or sometimes both [2]. Both the plant and controller models may be subjected to order reduction. For a plant with reduced order, the controller obtained is relatively of reduced order. However, its applications are restricted to low performance and cheap scenarios. For critical applications like avionics, medical etc., superior demands of accuracy results in a high order controller. The controller can then be reduced for easier realization and analysis [3].

This paper proposes a technique that relies on dimensionless representation and pole-zero dominance. Simultaneous consideration of both these concepts have not been much explored in control community in terms of model order reduction. In literature, the pole-zero dominance method does not cater for systems with poles at origin. In this work, a new method with some modifications is proposed to cater for this limitation. Simulation results highlight the performance of the proposed technique. Also, MATLAB has no toolbox related to dimensionless representation and the present work contributes towards this toolbox. The nomenclature of various symbols mentioned in this paper is presented in Table I.

TABLE I. NOMENCLATURE

\begin{tabular}{|c|c|}
\hline Symbol & Description \\
\hline$\sigma_{\mathrm{H}}$ & Hankel singular values \\
\hline $\mathbf{W}_{\mathbf{c}}$ & Controllability Gramian \\
\hline $\mathbf{W}_{\mathbf{0}}$ & Observability Gramian \\
\hline $\mathbf{s}_{i}$ & $\begin{array}{l}\text { Pole value of pole } \boldsymbol{p}_{\boldsymbol{i}} \text { where } \mathrm{i}=1, \ldots, \mathrm{n} \text { and } \mathrm{n}=\text { number of } \\
\text { poles }\end{array}$ \\
\hline$p_{n}$ & Pole with the largest real part \\
\hline$r_{p p i}$ & $\begin{array}{l}\text { Pole-to-pole dominance ratio of pole, } s_{i} \text {, with respect to } \\
\text { pole, } \mathbf{s}_{\mathbf{i}+\mathbf{1}}\end{array}$ \\
\hline$r_{p z i}$ & Pole-zero ratio of zero $\boldsymbol{z}_{\boldsymbol{i}}$ \\
\hline$r_{p p \min }$ & $\begin{array}{l}\text { Minimum value of pole-to-pole ratio below which the } \\
\text { degree of dominance is deemed to be insufficient }\end{array}$ \\
\hline$r_{p z \min }$ & Minimum pole-to-zero dominance ratio \\
\hline $\mathbf{T}_{\mathbf{p i}}$ & $\begin{array}{l}\qquad \mathbf{T}_{\mathbf{p i}}=\mathbf{1} / \mathbf{R e}\left(\boldsymbol{s}_{\boldsymbol{i}}\right) \\
\text { Time constant of pole } \boldsymbol{p}_{\boldsymbol{i}} \text {, with } \mathbf{s}_{\boldsymbol{i}} \text { being the pole value }\end{array}$ \\
\hline
\end{tabular}

\section{LITERATURE REVIEW}

Various methods have been proposed for model order reduction, most of which are based on Hankel singular values. For a Multi Input Multi Output (MIMO) Linear Time Invariant (LTI) system given in standard state-space form (with A, B, C and $\mathrm{D}$ matrices), Hankel singular values are given in Eq. 1 [4]. Here, the importance of a singular value is w.r.t. controllability and observability, given by Eq. 2 and Eq. 3 respectively [5].

$$
\begin{gathered}
\sigma_{\mathrm{H}}=\sqrt{\lambda_{\mathrm{i}}\left(\mathrm{W}_{\mathrm{c}} \mathrm{W}_{\mathrm{o}}\right)} \\
\mathrm{W}_{\mathrm{c}}=\int_{0}^{\infty} \mathrm{e}^{\mathrm{A} \tau} \mathrm{BB}^{\mathrm{T}} \mathrm{e}^{\mathrm{A}^{\mathrm{T}} \tau} \mathrm{d} \tau \\
\mathrm{W}_{\mathrm{o}}=\int_{0}^{\infty} \mathrm{e}^{\mathrm{A}^{\mathrm{T}} \tau} \mathrm{C}^{\mathrm{T}} \mathrm{Ce}^{\mathrm{A} \tau} \mathrm{d} \tau
\end{gathered}
$$

where, $\mathrm{W}_{\mathrm{c}}$ and $\mathrm{W}_{\mathrm{o}}$ satisfy the Lyapunov equation(s). 
The most commonly used model order reduction technique in theory is balanced truncation presented by Moore [6]. This technique uses balanced realization in which a minimal realization state-space is obtained. The system can then be partitioned into subsystems as shown in Eq. 4.

$$
\begin{gathered}
A=\left[\begin{array}{ll}
A_{11} & A_{12} \\
A_{21} & A_{22}
\end{array}\right], B=\left[\begin{array}{l}
B_{1} \\
B_{2}
\end{array}\right], \\
C=\left[\begin{array}{ll}
C_{1} & C_{2}
\end{array}\right], D=0
\end{gathered}
$$

Moore suggested that for the given balanced system, the subsystem $\left(A_{11}, B_{1}, C_{1}\right)$ should be a good enough approximation when the impulse response of this subsystem is same as that of the full model. However, the balanced truncation method gives zero error at high frequencies and nonzero error at low frequencies. To cater for this limitation, Yi and Brian [7] proposed a technique based on singular perturbation approximation which gives smaller errors at low frequencies and large errors at high frequencies.

Safonov and Chiang [4] stated balanced truncation as being badly conditioned. Numerical difficulties occur in calculating the transformation matrix when one or few of the modes are very nearly uncontrollable and unobservable. To cater these difficulties, the authors propose reduction via projections which are arbitrary bases for Eigen-spaces. For Eigen-spaces, two methods can be used: Schur decomposition of the product of the controllability and observability gramians [4] and another method based on Cholesky factors of the gramians [8].

Choroszucha et al. [9] investigated a new method using Schur decomposition by combining two existing methods: Balanced truncation and Modal truncation. The proposed technique gives better result than the 'reduce' command in MATLAB for reducing model order of two or less.

Furthermore, Poonam and Patil [10] presented a new method named Balanced Truncation Approximation (BTA) using bilinear conformal transformation approach. This method provides an error bound. Moreover, one of the conventional methods of model order reduction is based on modal approximation in which the original system is projected onto the Eigen-spaces which correspond to the few selected Eigenvalues. Benner et al. [11] selected dominant poles to project the system onto left and right Eigen-spaces for which the twosided Jacobi-Davidson method was chosen due to its robustness. Further details are reported in $[12,13]$.

\section{BACKGROUND}

\section{A. Dimensionless Representation}

The advantages of dimensionless representation w.r.t. model order reduction include; (i) It minimizes the number of parameters necessary for the system description. (ii) It may provide further knowledge of the parameters influence on the dynamical response. (iii) It gives better identification of asymptotic values whether they are too small or too large. These advantages provide a basis for selecting the parameters that may be truncated. The resultant reduced order model is then obtained by eliminating these parameters. For dimensional analysis, Buckingham $\mathrm{Pi}$ theorem works on the principle of independence of fundamental dimensions. The theorem does not explicitly consider poles and zeros but it can be applied to transfer function and state-space models. For mathematical details and procedure to obtain a dimensionless based representation model, the reader is referred to [14].

\section{B. Pole-Zero Dominance}

In some cases, it is possible to identify the set of dominant poles and/or zeros that has a relatively higher influence over the system dynamics compared to other poles/zeros. By considering only these dominant sets of poles and zeros, a transfer function of the system can be obtained with almost the same dynamic response. The pole and zero dominance can be computed based on two parameters; pole-to-pole dominance ratio and pole-to-zero dominance ratio [15].

1) Pole-to-pole dominance ratio: This ratio enables to determine the influence of one pole (or a group of poles) on the closed loop dynamic performance to be compared with that of another pole or group of poles [14]. Firstly, the poles are arranged in increasing magnitude of their real parts and subsequently with reduced time constants.

$\left|\operatorname{Re}\left(s_{1}\right)\right| \leq\left|\operatorname{Re}\left(s_{2}\right)\right| \leq \cdots \leq\left|\operatorname{Re}\left(s_{n}\right)\right|$

$\mathrm{T}_{\mathrm{p} 1} \geq \mathrm{T}_{\mathrm{p} 2} \geq \cdots \geq \mathrm{T}_{\mathrm{pn}}$

The poles according to Eq. 5 and Eq. 6 are arranged in decreasing degree of dominance. Next, pole-to-pole ratio is determined for each pole with the previous pole in line as per the sequence defined in Eq. 5. This ratio is given by:

$\mathrm{r}_{\mathrm{pp} \mathrm{i}}=\operatorname{Re}\left(\mathrm{s}_{\mathrm{i}+1}\right) / \operatorname{Re}\left(\mathrm{s}_{\mathrm{i}}\right)$

The method to determine the dominant poles for reduced order model is as follows:

for $i=1,2, \cdots, n: r_{p p i}=\operatorname{Re}\left(\mathrm{s}_{\mathrm{i}+1}\right) / \operatorname{Re}\left(\mathrm{s}_{\mathrm{i}}\right)=T_{p i} / T_{p i+1}$

if $r_{p p i} \leq r_{p p \min }:$ keep $\mathrm{s}_{\mathrm{i}+1}$, else selection is complete.

For ratio below $r_{p p \text { min }}$, the degree of dominance is considered to be deficient for computing the reduced model.

2) Pole-to-zero dominance ratio: This ratio enables assessment of the effect of a pole (or a group of poles) closer to the origin of the s-plane than a zero (or a group of zeros) in reducing the influence of that zero (or a group of zeros) on the closed loop dynamic performance [15]. The effect of zeros on the control system performance is greater with decreasing magnitudes of the zeros. So, similar to poles, the zeros are arranged in increasing order of their magnitudes as given in Eq. 8 .

$$
\left|\mathrm{z}_{1}\right| \leq\left|\mathrm{z}_{2}\right| \leq \cdots \leq\left|\mathrm{z}_{\mathrm{m}}\right|
$$

The pole-to-zero dominance ratio is given as in Eq. 9.

$$
r_{p z}=\left|z_{i}\right| / \operatorname{Re}\left(p_{n}\right), i=1,2, \cdots, m
$$


where, $m$ is the number of zeros and $p_{n}$ is the pole with the largest real part. Thus, for each zero, it gives the smallest value of $r_{p z}$. The dominant zeros can be determined using a minimum $r_{p z}$ value $\left(r_{p z \text { min }}\right)$. The zeros for which $r_{p z} \leq$ $r_{p z \text { min }}$, are considered as dominant zeros. These dominant zeros are considered while computing the reduced system.

\section{EXISTING APPROACHES AND PROPOSED METHOD}

This research investigates pole dominance based model order reduction and the augmentation of dimensionless representation with the existing reduction techniques based on Hankel singular values. Dimensionless representation can be performed either before or after applying Hankel singular values based reduction techniques.

\section{A. Limitations of Dimensionless Representation}

The dimensionless representation of a system can be obtained through non-dimensionalizing the transfer function or the state-space model of the system. Considering state-space model in MATLAB, the stability of the system after nondimensionalizing is not always guaranteed. Thus, we cannot apply the Hankel singular value based built-in methods in MATLAB for reduction. Consider an example of a plant model given below with three inputs, three outputs and five states.

$$
\begin{aligned}
& A=\left[\begin{array}{ccccc}
0 & 0 & 1.132 & 0 & -1 \\
0 & -0.0538 & -0.1712 & 0 & 0.0705 \\
0 & 0 & 0 & 1 & 0 \\
0 & 0.0485 & 0 & -0.8556 & -1.013 \\
0 & -0.2909 & 0 & 1.0532 & -0.6859
\end{array}\right], \\
& B=\left[\begin{array}{ccc}
0 & 0 & 0 \\
-0.12 & 1 & 0 \\
0 & 0 & 0 \\
4.419 & 0 & -1.665 \\
1.575 & 0 & -0.0732
\end{array}\right] \\
& C=\left[\begin{array}{lllll}
1 & 0 & 0 & 0 & 0 \\
0 & 1 & 0 & 0 & 0 \\
0 & 0 & 1 & 0 & 0
\end{array}\right], D=\left[\begin{array}{lll}
0 & 0 & 0 \\
0 & 0 & 0 \\
0 & 0 & 0
\end{array}\right]
\end{aligned}
$$

The poles of the original plant model are: $0.000+0.000 \mathrm{i},-$ $0.780+1.030 \mathrm{i},-0.780-1.030 \mathrm{i},-0.018+0.183 \mathrm{i},-0.018-$ 0.183 i. All the poles are in the Left Half Plane (LHP) as depicted in Figure 1. Therefore, the system is stable.

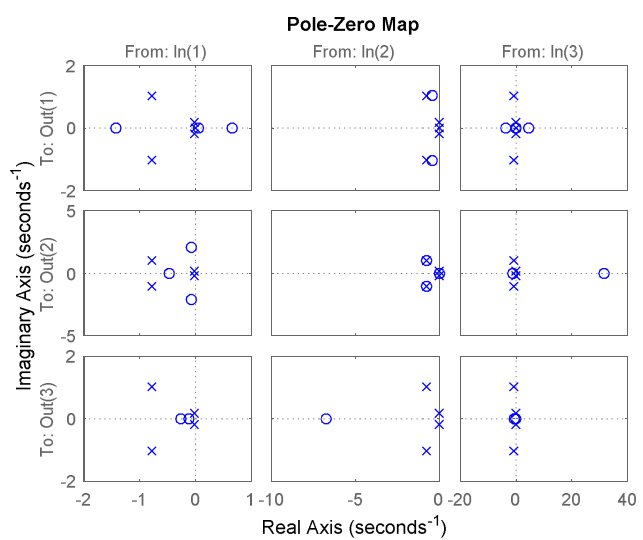

Fig. 1. Pole-Zero map of the Original System
All the poles have been shifted to the Right Half Plane (RHP) thus, making the system unstable as shown in Figure 2.

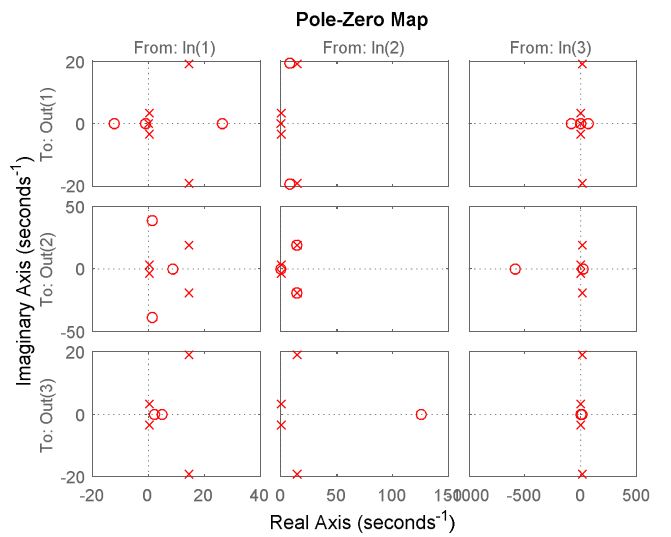

Fig. 2. Pole-Zero map of the Dimensionless System

Considering the dimensionless transfer function, according to the steps laid out in Buckingham Pi theorem, the dominant time constant $\left(\tau_{\mathrm{p} 1}\right)$ is chosen as a first step in computing the dimensionless transfer function for a given transfer function in the generic pole-zero form (Eq. 10). Each parameter of the transfer function is then divided by $\tau_{\mathrm{p} 1}$. The problem is encountered in the case when the pole(s) are complex. Buckingham Pi theorem does not mention anything related to complex poles. Neglecting the imaginary part results in changing the system modes altogether. Thus, this proves to be a limitation that dimensionless representation of transfer function can only be applied to non-oscillatory systems.

$G(s)=k \frac{\prod_{j=1}^{m}\left(\tau_{c j} s+1\right)}{s^{l} \prod_{i=1}^{n}\left(\tau_{p i} s+1\right)} e^{-h s}$

\section{B. Limitations of Pole-Zero Dominance}

Pole-zero dominance helps to find the dominant poles and dominant zeros. However, the pole-to-pole ratio is given for a zero-less system and is applied without considering the zeros of the system. Similarly, the pole-to-zero ratio is given with the poles considered as constant. Also, this method does not take into account the pole/poles at the origin since the minimum pole-to-pole ratio and minimum pole-to-zero ratio for evaluating dominance of poles and zeros respectively are provided for systems having no pole/poles at origin. However, this is not the case for many systems in the field of controls.

\section{Proposed Method}

For a system with real poles, dimensionless representation of transfer function is considered. Two cases are considered; pole-zero cancellation and elimination of slow mode. The cancellation is done for exact pole-zero match or when the pole and zero are in close proximity. The range is set according to the user's choice. If cancellation is possible, then the method reduces the number of poles and number of zeros by one. Then the method moves onto next iteration for the next pole. If the cancellation is possible for a pole with any already eliminated zero or vice versa, then the difference between the two possible 
cancellations is considered. The pole-zero pair with more nearness in range to each other is selected for cancellation and is then eliminated. Next, the model is checked for any slow modes. As the parameters representing the poles $\left(\overline{\boldsymbol{\tau}}_{\boldsymbol{p} \boldsymbol{i}}\right)$ and zeros $\left(\overline{\boldsymbol{\tau}}_{\boldsymbol{c} j}\right)$ are non-dimensionalized, where $\mathrm{i}=1, \ldots, \mathrm{n}, \mathrm{j}=1, \ldots, \mathrm{m}$, the smallness of the parameter can be easily determined. The choice of threshold on parameter value so as to be considered as slow (and thus eliminated) is arbitrary. This threshold is referred as boundary value in the present discussion. Then, in each iteration, the value of dimensionless parameter $\overline{\boldsymbol{\tau}}_{\boldsymbol{p} \boldsymbol{i}}$ is checked against the boundary value. If the value of $\overline{\boldsymbol{\tau}}_{\boldsymbol{p} \boldsymbol{i}}$ is smaller than the boundary value, the respective pole and remaining poles corresponding to the parameters with value less than the boundary value are eliminated i.e.

if $\overline{\boldsymbol{\tau}}_{\boldsymbol{p} \boldsymbol{i}}>$ boundary value, keep pole $p_{i}$

else eliminate $p_{i}, p_{i+1}, \cdots, p_{n}$

As a result, reduced number of dimensionless parameters representing reduced number of poles and zeros are obtained. Finally, these parameters are dimensionalized to pole and zero form, based on which, the reduced system model is obtained.

In pole-zero dominance, any poles located at the origin are not considered in evaluation of pole-to-pole dominance. This is catered by dividing the system into two subsystems; system with poles at origin and system with remaining poles. The dominant poles and zeros are computed for the remaining subsystem without considering poles at origin. After determination of the dominant poles and zeros, the rest of the poles and zeros of this subsystem are eliminated. Finally, the poles and zeros of the two subsystems are combined. The flow chart of proposed method is illustrated in Figure 3.

\section{SiMULATION RESULTS}

Simulations were performed on MATLAB (R2015b) using 64-bit Windows 10 Operating system installed on a $3.1 \mathrm{GHz}$ core i-7 CPU and with a RAM of $8 \mathrm{~GB}$. Simulation were performed on oscillatory and non-oscillatory models with different orders. First, the models were tested for dimensionless representation based reduction and their results were noted and compared to the expected outcome. The DC gain of the original and the reduced model were normalized to unity for comparison purposes. If the system has oscillatory modes, then dimensionless representation method is not applicable. For non-oscillatory systems, the method is applied and possible reduction is done based on dimensionless representation. After reduction of parameters, the model is taken back to its original dimensional form and is then compared to the original model.

Secondly, the models were tested for pole-zero dominance based reduction for oscillatory class of the systems. The poles and zeros of the model were obtained and then the algorithm for pole-zero dominance was applied. Normalizing the DC gains to unity, the error between the step responses of the original and the reduced model was computed for an interval of $10 \mu \mathrm{sec}$. up to $100 \mathrm{sec}$. Thus, the computed percentage error gives good accuracy.

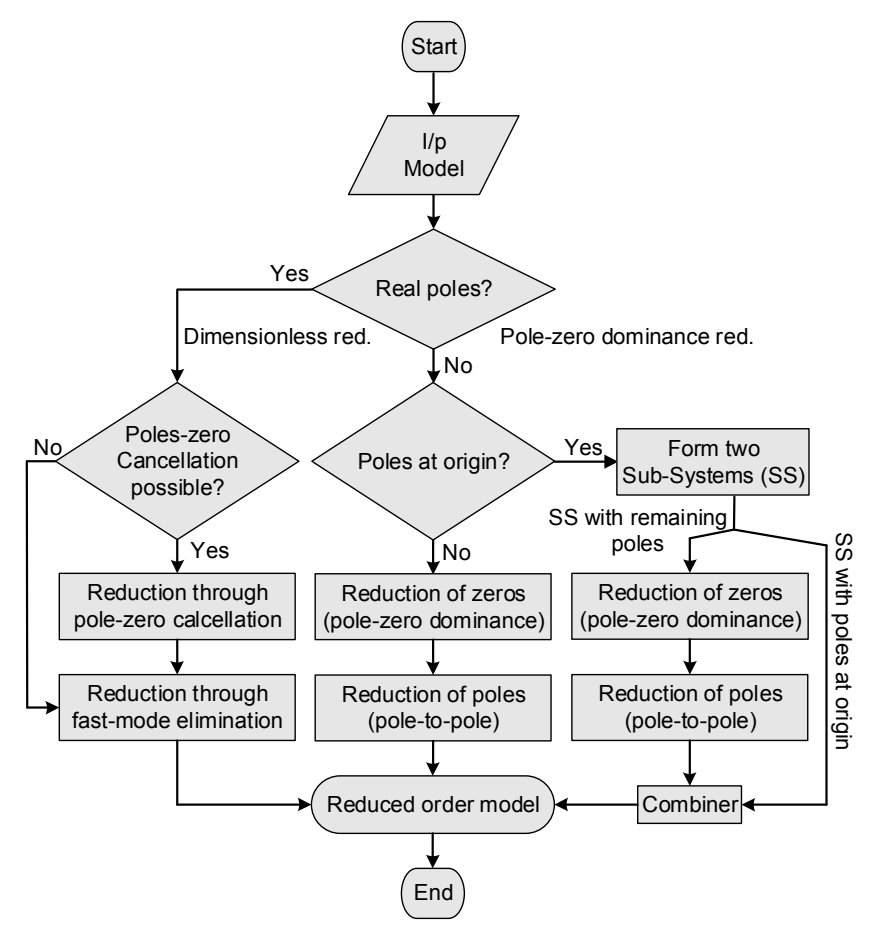

Fig. 3. Flow chart of proposed method for model order reduction

A. Example 1: Dimensionless Representation based Reduction Through pole-zero Cancellation

Consider a typical system with transfer function as

$Y(s)=\frac{1.02 s+1.02}{0.34 s^{3}+10.57 s^{2}+11.25 s+1.02}$

The poles are located at $-0.1,-1,-30$ while a zero is found at on: -1 . The proposed method reduced the model order and the resulting reduced transfer function is written as,

$Y_{\text {red }}(s)=\frac{1.02}{0.34 s^{2}+10.23 s+1.02}$

As expected, the algorithm reduces the model based on pole-zero cancellation at -1 . The percentage error is $0.000775 \%$ and it can be seen in Figure 4 that the reduced model closely follows the original model.

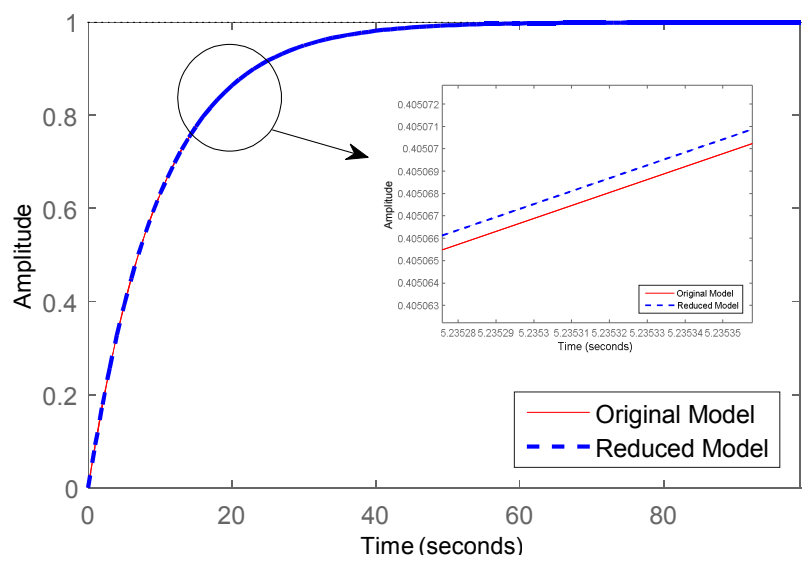

Fig. 4. Step responses of the original and the system reduced through pole-zero cancellation. 
B. Example 2: Dimensionless Representation based Reduction through Fast-mode Elimination

Considering another system with transfer function as,

$$
Y(s)=\frac{s+2}{s^{3}+101 s^{2}+101 s+1}
$$

The poles of the system are located at $-0.01,-1,-100$ while a zero is found at -1 . The proposed method reduced the model order and the resulting reduced transfer function is given as,

$$
Y_{\text {red }}(s)=\frac{2 s+2.01}{201 s^{2}+201 s+2.01}
$$

As expected, the pole $\mathrm{p}=-100$ is eliminated as we may disregard fast model dynamics. As can be seen in the plot of Hankel singular value (Figure 5), the major contribution in the system dynamics is from the first pole and least contribution is from the pole located at $\mathrm{p}=-100$. The percentage error between the original and the reduced model is $0.48202 \%$.

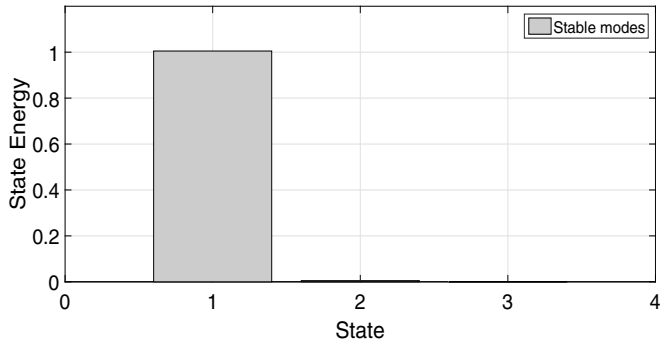

Fig. 5. HSV plot showing the contribution of the poles of the original system to the system dynamics.

\section{Example 3: Pole-zero Dominance based Reduction}

Considering a system with transfer function as,

$Y(s)=\frac{s+2}{0.2564 s^{4}+7.999 s^{3}+9.348 s^{2}+3.605 s+2}$

The poles are located at $-0.01-0.5 \mathrm{i},-0.01+0.5 \mathrm{i},-1,-30$ while a zero is found at -2 . The transfer function of the reduced order system is found to be:

$Y_{\text {red }}(s)=\frac{3.9 s+7.8}{30 s^{3}+36 s^{2}+13.8 s+7.8}$

The Hankel singular values of the given system are shown in Figure 6. It can be seen that the pole at -30 has very less contribution as the value of its time constant is very small compared to the previous pole located at $p=-1$. Thus, the pole at -30 can be eliminated.

The percentage error between the step responses of the original model and reduced model is found to be $1.1884 \%$ which is less than $5 \%$, as expected. The difference between the original and the reduced models is evident from bode plot shown in Figure 7. At low and medium frequencies, the reduced model matches the original model. Slight deviation is seen at higher frequencies.

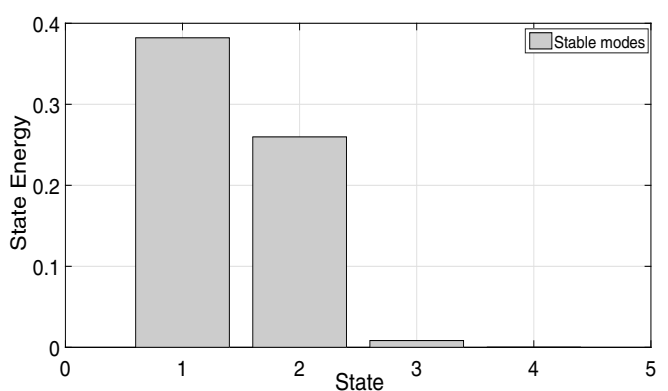

Fig. 6. HSV plot showing the contribution of the poles of the original system to the system dynamics.

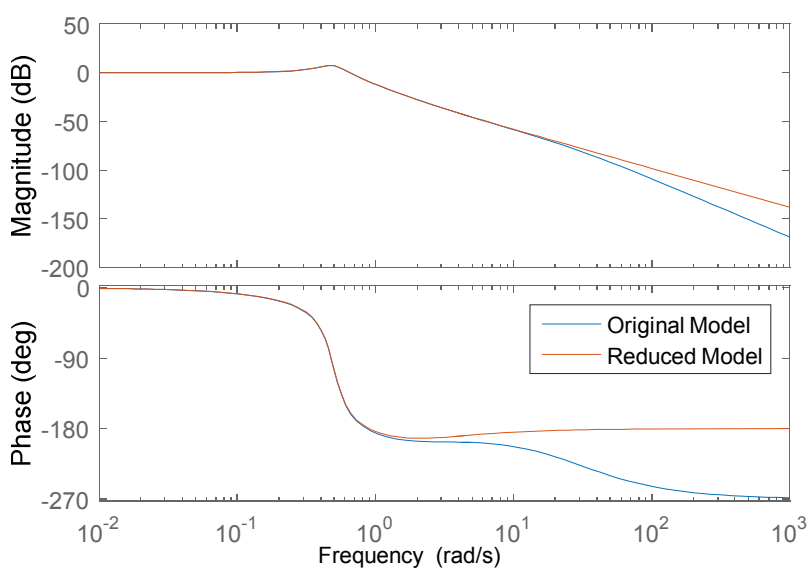

Fig. 7. Bode Diagram of the original and the reduced model.

D. Example 4: Augmentation of Dimensionless Representation and Hankel Singular Values based Model Order Reduction Considering a $5^{\text {th }}$ order system with transfer function as,

$$
Y(s)=\frac{s^{3}+11 s^{2}+36 s+36}{6 s^{5}+639.1 s^{4}+3976 s^{3}+6976 s^{2}+3669 s+36}
$$

The poles are located at $-0.01,-1,-1.5,-4,-100$ while zeros are found at $-2,-3,-6$. The transfer function of the reduced system through dimensionless representation based reduction is determined as

$$
Y(s)=\frac{6 s^{3}+39.05 s^{2}+69.29 s+36.33}{605.6 s^{4}+3942 s^{3}+7004 s^{2}+3703 s+36.33}
$$

The percentage error between the reduced and original system is $0.84735 \%$. The system is further reduced using HSV based method using MATLAB command 'reduce'. The system is reduced to $3^{\text {rd }}$ order from $4^{\text {th }}$ order. The percentage error is $0.8743492 \%$. The reduced transfer function is given by

$$
Y_{\mathrm{r}}(s)=\frac{0.009908 s^{2}+0.02301 s+0.0132}{s^{3}+2.324 s^{2}+1.343 s+0.0132}
$$


Finally, the original model $\mathrm{Y}(\mathrm{s})$ was reduced to $3^{\text {rd }}$ order system $\mathrm{Y}_{\text {red }}(\mathrm{s})$ using MATLAB 'reduce' command. Results are then compared with the proposed augmented method.

$$
Y_{\text {red }}(s)=\frac{0.00151 s^{2}+0.00977 s+0.004766}{s^{3}+1.428 s^{2}+0.4908 s+0.004766}
$$

The percentage error for this reduction is $0.0016 \%$ which is very less than the percentage error in the reduction performed by the augmented method. Thus, the augmented method does not give better results compared to Hankel singular values based reduction methods. However, it can identify any polezero cancellation and slow modes to be reduced. The Hankel singular values based methods do not explicitly check for polezero cancellation and slow-modes. Furthermore, the augmented method ensures the error to be less than or equal to $5 \%$.

\section{CONCLUSION}

In recent years, numerous research works have been reported in the domain of model order reduction with an attempt to improve the existing methods. Dimensionless representation in model order reduction is a new field which is investigated in the present work. The proposed approach provides reduction based on the smallness or largeness of the value of the dimensionless parameter. The relativeness in the value of the dimensionless parameter makes the analysis of system dynamics easier. However, dimensionless representation is restricted to be applicable only for nonoscillatory systems. To deal with oscillatory systems, pole-zero dominance is considered. Once the dominant poles and dominant zeros are computed, the system can be reduced by eliminating the non-dominant poles and non-dominant zeros. The method ensures that the error between step responses of the reduced and the original models is always less than $5 \%$.

Dimensionless representation is a very powerful tool, which can be further explored to attain the maximum advantages in terms of model order reduction. For systems with complex poles, this domain could be further explored and a method may be devised for reduction through dimensionless representation. Secondly, pole-zero dominance method only considers the ratio between two consecutive poles. Another dimension that can be investigated in pole-zero dominance method is the consideration of controllability and observability of the non-dominant group identified.

\section{REFERENCES}

[1] J. Iqbal, M. Ullah, S. G. Khan, B. Khelifa, and S. Ćuković, "Nonlinear control systems-A brief overview of historical and recent advances," Nonlinear Engineering, 2017, DOI: 10.515/nleng2016-0077.

[2] O. Khan, M. Pervaiz, E. Ahmad, and J. Iqbal, "On the derivation of novel model and sophisticated control of flexible joint manipulator," Revue Roumaine des Sciences Techniques-Serie Electrotechnique et Energetique, vol. 62, pp. 103-108, 2017.
[3] Z. Bai, "Krylov subspace techniques for reducedorder modeling of large-scale dynamical systems," Applied numerical mathematics, vol. 43, pp. 9-44, 2002.

M. G. Safonov and R. Chiang, "A Schur method for balanced-truncation model reduction," IEEE Transactions on Automatic Control, vol. 34, pp. 729733, 1989.

[5] K. Willcox and J. Peraire, "Balanced model reduction via the proper orthogonal decomposition," AIAA journal, vol. 40, pp. 2323-2330, 2002.

[6] B. Moore, "Principal component analysis in linear systems: Controllability, observability, and model reduction," IEEE transactions on automatic control, vol. 26, pp. 17-32, 1981.

[7] Y. Liu and B. D. Anderson, "Singular perturbation approximation of balanced systems," International Journal of Control, vol. 50, pp. 1379-1405, 1989.

[8] S. J. Hammarling, "Numerical solution of the stable, non-negative definite lyapunov equation lyapunov equation," IMA Journal of Numerical Analysis, vol. 2, pp. 303-323, 1982.

[9] R. Choroszucha, J. Sun, and K. Butts, "Closed-loop model order reduction and MPC for diesel engine airpath control," in American Control Conference (ACC), 2015, 2015, pp. 3279-3284.

[10] P. J. Patil and M. D. Patil, "Model order reduction of high order LTI system using balanced truncation approximation," in Process Automation, Control and Computing (PACC), 2011 International Conference on, 2011, pp. 1-6.

[11] P. Benner, M. E. Hochstenbach, and P. Kurschner, "Model order reduction of large-scale dynamical systems with Jacobi-Davidson style eigensolvers," in Communications, Computing and Control Applications (CCCA), 2011 International Conference on, 2011, pp. 1-6.

[12] A. Stathopoulos, "A Case for a Biorthogonal Jacobi-Davidson Method: Restarting and Correction Equation," SIAM Journal on Matrix Analysis and Applications, vol. 24, pp. 238-259, 2002.

[13] M. E. Hochstenbach and G. L. Sleijpen, "Two-sided and alternating Jacobi-Davidson," Linear Algebra and its Applications, vol. 358, pp. 145-172, 2003.

[14] P. Balaguer, Application of dimensional analysis in systems modeling and control design: The Institution of Engineering and Technology, 2013.

[15] S. J. Dodds, "Motion Control," in Feedback Control, ed: Springer, 2015, pp. 793-846. 